\title{
On weak solutions to a generalized Camassa-Holm equation with solitary wave
}

\section{Yunxi Guo ${ }^{1 *}$}

\section{"Correspondence:}

matyunxiguo@126.com

'Department of Mathematics, Zunyi

Normal University, Zunyi, China

\begin{abstract}
A generalized Camassa-Holm equation proposed by Novikov is considered. The existence and uniqueness of a positive weak solution for the equation is established by using a classical method.
\end{abstract}

MSC: 35D05; 35G25; 35L05; 35Q35

Keywords: Existence; Weak solution; A generalized Camassa-Holm equation

\section{Introduction}

Recently, Novikov [22] proposed the following integrable quasi-linear scalar evolution equation of order 2 :

$$
\left(1-\epsilon^{2} \partial_{x}^{2}\right) u_{t}=\partial_{x}\left(2-\epsilon \partial_{x}\right)\left(1+\epsilon \partial_{x}\right) u^{2}
$$

where $\epsilon \neq 0$ is a real constant.

Letting $v(t, x)=u(\epsilon t, \epsilon x)$, one can transform Eq. (1) into the following form:

$$
u_{t}-u_{t x x}=\partial_{x}\left(2-\partial_{x}\right)\left(1+\partial_{x}\right) u^{2}
$$

It was shown in [22] that Eq. (2) possesses a hierarchy of local higher symmetries. Equation (2) is regarded as a generalized Camassa-Holm equation [22]. In [17], Li and Yin establish the local existence and uniqueness of strong solutions for Eq. (2) in nonhomogeneous Besov spaces by using the Littlewood-Paley theory. Under some assumptions, a blowup criterion and a global existence result for the equation are also presented in [17]. The well-posedness of (2) is studied in [11] for the periodic and the nonperiodic cases in the sense of Hadamard. In addition, nonuniform dependence is proved by using the method of approximate solutions and well-posedness estimates. To the best of our knowledge, up to now the weak solutions for Eq. (2) have not been investigated yet.

The equation closest to the relevant problem (2) is the Degasperis-Procesi equation,

$$
\left(1-\partial_{x}^{2}\right) u_{t}=-4 u u_{x}+3 u_{x} u_{x x}+u u_{x x x}
$$

(c) The Author(s) 2020. This article is licensed under a Creative Commons Attribution 4.0 International License, which permits use, sharing, adaptation, distribution and reproduction in any medium or format, as long as you give appropriate credit to the original author(s) and the source, provide a link to the Creative Commons licence, and indicate if changes were made. The images or other third party material in this article are included in the article's Creative Commons licence, unless indicated otherwise in a credit line to the material. If material is not included in the article's Creative Commons licence and your intended use is not permitted by statutory regulation or exceeds the permitted use, you will need to obtain permission directly from the copyright holder. To view a copy of this licence, visit http://creativecommons.org/licenses/by/4.0/. 
Degasperis, Holm and Hone [12] proved the formal integrability of Eq. (2) by constructing a Lax pair. They showed that it has a bi-Hamiltonian structure and there is an infinite sequence of conserved quantities. Since the Degasperis-Procesi equation was born, much attention has been attracted by the study its dynamics. Yin proved local well-posedness of Eq. (2) on the line [24] and on the circle [25]. In addition, the precise blow-up scenario and blow-up structure for the equation were derived in [24, 25]. Lenells [16] classified all weak traveling wave solutions. Matsuno [20] obtained multisolutions of Eq. (2). Escher et al. [13] investigated the blow-up phenomena and global weak solutions for Degasperis-Procesi equation. In a different direction, Coclite and Karlsen [3-5], and Lundmark [19] initiated a study of discontinuous solutions (shock wave) to the Degasperis-Procesi equation (2). It is shown in [2] that a new blow-up quantity along the characteristics is established for the Degasperis-Procesi equation (2). The other equations related to Eq. (2), such as the Camassa-Holm equation, the Novikov equation and the Modified Camassa-Holm equation with cubic nonlinearity, can be found in $[1,6-10,14,15,18,22,23]$ and the references therein.

Inspired by the ideas from $[13,26]$, in this paper, we investigate the weak solutions for the following Cauchy problem:

$$
\left\{\begin{array}{l}
u_{t}-u_{t x x}=\partial_{x}\left(2-\partial_{x}\right)\left(1+\partial_{x}\right) u^{2} \\
u(0, x)=u_{0}(x)
\end{array}\right.
$$

More precisely, we focus on the existence and uniqueness of positive weak solutions to the problem (4) using the method from [11] under the condition $y_{0}=u_{0}-u_{0 x x} \in \mathcal{M}^{+}$. One of the difficult issues in our proof is how to prove that there is a subsequence of $\left\{u^{n}\right\}$ which converges pointwise a.e. to a function $u \in H_{\text {loc }}^{1}\left(\mathbb{R}_{+} \times \mathbb{R}\right)$ that satisfies (4) in the sense of distributions, and how to show that $u \in C_{w}\left(\mathbb{R}_{+} ; H^{1}(\mathbb{R})\right)$, the space of continuous functions from $\mathbb{R}_{+}$with values in $H^{1}(\mathbb{R})$ when the latter space is equipped with its weak topology. Luckily, using $y_{0}=u_{0}-u_{0 x x}>0$ and the estimate $\|u(t, \cdot)\|_{L^{\infty}(\mathbb{R})} \leq \frac{3}{2}\left\|u_{0}\right\|_{L^{3}(\mathbb{R})}^{2} t+\left\|u_{0}\right\|_{L^{\infty}}$, we successfully overcome the problems.

Notations The space of all infinitely differentiable functions $\phi(t, x)$ with compact support in $[0,+\infty) \times \mathbb{R}$ is denoted by $C_{0}^{\infty}$. Let $1 \leq p<+\infty$ and $L^{p}=L^{p}(\mathbb{R})$ be the space of all measurable functions $h(t, x)$ such that $\|h\|_{L^{P}}^{P}=\int_{\mathbb{R}}|h(t, x)|^{p} d x<\infty$. We define $L^{\infty}=L^{\infty}(\mathbb{R})$ with the standard norm $\|h\|_{L^{\infty}}=\inf _{m(e)=0} \sup _{x \in \mathbb{R} \backslash e}|h(t, x)|$. For any real number $s$, let $H^{s}=H^{s}(\mathbb{R})$ denote the Sobolev space with the norm defined by $\|h\|_{H^{s}}=\left(\int_{\mathbb{R}}\left(1+|\xi|^{2}\right)^{s}|\hat{h}(t, \xi)|^{2} d \xi\right)^{\frac{1}{2}}<\infty$, where $\hat{h}(t, \xi)=\int_{\mathbb{R}} e^{-i x \xi} h(t, x) d x$.

We denote by $*$ the convolution. Let $\|\cdot\|_{X}$ denote the norm of Banach space $X$ and $\langle\cdot, \cdot\rangle$ denote the $H^{1}(\mathbb{R}), H^{-1}(\mathbb{R})$ duality bracket. Let $\mathcal{M}(\mathbb{R})$ be the space of Radon measures on $\mathbb{R}$ with bounded total variation and $\mathcal{M}^{+}(\mathbb{R})$ be the subset of positive measures. Finally, we write $\mathrm{BV}(\mathbb{R})$ for the space of functions with bounded variation, $V(f)$ being the total variation of $f \in \mathrm{BV}(\mathbb{R})$.

\section{Preliminaries}

Throughout this paper, let $\left\{\rho_{n}\right\}_{n \geq 1}$ denote the mollifiers

$$
\rho_{n}(x):=\left(\int_{\mathbb{R}} \rho(\xi) d \xi\right)^{-1} n \rho(n x), \quad x \in \mathbb{R}, n \geq 1,
$$


where $\rho \in C_{c}^{\infty}(\mathbb{R})$ is defined by

$$
\rho(x):= \begin{cases}e^{\frac{1}{x^{2}-1}} & \text { for }|x|<1 \\ 0 & \text { for }|x| \geq 1\end{cases}
$$

Thus, we get

$$
\int_{\mathbb{R}} \rho_{n}(x) d x=1, \quad \rho_{n} \geq 0, x \in \mathbb{R}, n \geq 1
$$

Note that if $G(x):=\frac{1}{2} e^{-|x|}, x \in \mathbb{R}$. Then $\left(1-\partial_{x}^{2}\right)^{-1} f=G * f$ for all $f \in L^{2}(\mathbb{R})$ and $G *(u-$ $\left.u_{x x}\right)=u$. Using this identity, we rewrite problem (4) in the form

$$
\begin{cases}u_{t}-u_{t x x}-4 u u_{x}+6 u_{x} u_{x x}+2 u u_{x x x}=2 u_{x}^{2}+2 u u_{x x x}, & t>0, x \in \mathbb{R}, \\ u(0, x)=u_{0}(x), & x \in \mathbb{R},\end{cases}
$$

which is equivalent to

$$
\begin{cases}u_{t}-2 u u_{x}-\partial_{x} G *\left[u^{2}+\left(u^{2}\right)_{x}\right]=0, & t>0, x \in \mathbb{R}, \\ u(0, x)=u_{0}(x), & x \in \mathbb{R} .\end{cases}
$$

Next, we give some useful results.

Lemma 2.1 Let $f: \mathbb{R} \rightarrow \mathbb{R}$ be uniformly continuous and bounded. If $\mu \in \mathcal{M}(\mathbb{R})$, then

$$
\left[\rho_{n} *(f \mu)-\left(\rho_{n} * f\right)\left(\rho_{n} * \mu\right)\right] \underset{n \rightarrow \infty}{\longrightarrow} 0 \text { in } L^{1}(\mathbb{R})
$$

Lemma 2.2 Let $f: \mathbb{R} \rightarrow \mathbb{R}$ be uniformly continuous and bounded. If $g \in L^{\infty}(\mathbb{R})$, then

$$
\left[\rho_{n} *(f g)-\left(\rho_{n} * f\right)\left(\rho_{n} * g\right)\right] \underset{n \rightarrow \infty}{\longrightarrow} 0 \quad \text { in } L^{\infty}(\mathbb{R})
$$

Lemma 2.3 Let $T>0$. If $f, g \in L^{2}\left((0, T) ; H^{1}(\mathbb{R})\right)$ and $\frac{d f}{d t}, \frac{d g}{d t} \in L^{2}\left((0, T) ; H^{-1}(\mathbb{R})\right)$, then $f, g$ are a.e. equal to a function continuous from $[0, T]$ into $L^{2}(\mathbb{R})$ and

$$
\langle f(t), g(t)\rangle-\langle f(s), g(s)\rangle=\int_{s}^{t}\left\langle\frac{d(f(\tau))}{d \tau}, g(\tau)\right\rangle d \tau+\int_{s}^{t}\left\langle\frac{d(g(\tau))}{d \tau}, f(\tau)\right\rangle d \tau
$$

for all $s, t \in[0, T]$.

Lemma 2.4 Assume that $u(t, \cdot) \in W^{1,1}(\mathbb{R})$ is uniformly bounded in $W^{1,1}(\mathbb{R})$ for all $t \in \mathbb{R}_{+}$. Then for a.e. $t \in \mathbb{R}_{+}$

$$
\frac{d}{d t} \int_{\mathbb{R}}\left|\rho_{n} * u\right| d x=\int_{\mathbb{R}}\left(\rho_{n} * u_{t}\right) \operatorname{sgn}\left(\rho_{n} * u\right) d x
$$

and

$$
\frac{d}{d t} \int_{\mathbb{R}}\left|\rho_{n} * u_{x}\right| d x=\int_{\mathbb{R}}\left(\rho_{n} * u_{x t}\right) \operatorname{sgn}\left(\rho_{n} * u_{x}\right) d x
$$


Lemmas 2.1-2.4 can be found in [11].

\section{Global weak solution}

Lemma 3.1 Let $u_{0} \in L^{3}(\mathbb{R}) \cap H^{s}(\mathbb{R}), s>\frac{3}{2}$ and $y_{0}=\left(1-\partial_{x}^{2}\right) u_{0} \geq 0$ for all $x \in \mathbb{R}$. Then the solutions of problem (6) exist globally in time. Moreover, if $y(t, \cdot)=u-u_{x x}$, then, for all $t \in \mathbb{R}_{+}$

(i) $y(t, \cdot) \geq 0, u(t, \cdot) \geq 0,\left|u_{x}(t, \cdot)\right| \leq u(t, \cdot)$ and $\|u(t, \cdot)\|_{L^{\infty}(\mathbb{R})} \leq \frac{3}{2}\left\|u_{0}\right\|_{L^{3}(\mathbb{R})}^{2} t+\left\|u_{0}\right\|_{L^{\infty}}$ on $\mathbb{R}$.

(ii) $\|u\|_{H^{1}} \leq\left\|u_{0}\right\|_{H^{1}} \exp \left[\frac{3}{2}\left\|u_{0}\right\|_{L^{3}(\mathbb{R})}^{2} t^{2}+2\left\|u_{0}\right\|_{L^{\infty}} t\right]$.

Proof The proof of (i) may be found in [17]. Now, we prove (ii).

Multiplying the first equation of problem (6) by $u$ and integrating by parts, we find

$$
\begin{aligned}
\frac{1}{2} \frac{d}{d t} \int_{\mathbb{R}}\left(u^{2}+u_{x}^{2}\right) d x & =\int_{\mathbb{R}} u_{x}^{3} d x-\int_{\mathbb{R}} 2 u u_{x}^{2} d x, \\
& \leq 2\left\|u_{x}\right\|_{L^{\infty}} \int_{\mathbb{R}}\left(u^{2}+u_{x}^{2}\right) d x
\end{aligned}
$$

which yields

$$
\begin{aligned}
\|u\|_{H^{1}}^{2} & \leq\left\|u_{0}\right\|_{H^{1}}^{2} e^{4 \int_{0}^{t}\left\|u_{x}\right\|_{L^{\infty}} d \tau} \\
& \leq\left\|u_{0}\right\|_{H^{1}}^{2} \exp \left[3\left\|u_{0}\right\|_{L^{3}}^{2} t^{2}+4\left\|u_{0}\right\|_{L^{\infty}} t\right]
\end{aligned}
$$

where the Gronwall inequality and (i) were used. This proves (ii) and completes the proof of the lemma.

Theorem 3.1 Let $u_{0} \in H^{1}(\mathbb{R}) \cap L^{3}(\mathbb{R})$, and $y_{0}=\left(u_{0}-u_{0 x x}\right) \in \mathcal{M}^{+}(\mathbb{R})$. Then problem (6) has a unique solution $u \in W^{1, \infty}\left(\mathbb{R}_{+} \times \mathbb{R}\right) \cap L_{\text {loc }}^{\infty}\left(\mathbb{R}_{+} ; H^{1}(\mathbb{R})\right)$ with initial value $u_{0}$ and such that $\left(u-u_{x x}\right) \in \mathcal{M}^{+}$, a.e. $t \in \mathbb{R}_{+}$is uniformly bounded on $\mathbb{R}$.

Proof We split the proof of Theorem 3.1 in two parts.

Let $u_{0} \in H^{1}(\mathbb{R})$ and $y_{0}=u_{0}-u_{0, x x} \in \mathcal{M}^{+}(\mathbb{R})$. Note that $u_{0}=G * y_{0}$. Thus, for $\varphi \in L^{\infty}(\mathbb{R})$, we have

$$
\begin{aligned}
\left\|u_{0}\right\|_{L^{1}(\mathbb{R})} & =\left\|G * y_{0}\right\|_{L^{1}(\mathbb{R})}=\sup _{\|\varphi\|_{L^{\infty}(\mathbb{R}) \leq 1}} \int_{\mathbb{R}} \varphi(x)\left(G * y_{0}\right)(x) d x \\
& =\sup _{\|\varphi\|_{L^{\infty}(\mathbb{R})} \leq 1} \int_{\mathbb{R}} \varphi(x) \int_{\mathbb{R}} G(x-\xi) y_{0}(\xi) d \xi d x \\
& =\sup _{\|\varphi\|_{L^{\infty}(\mathbb{R})} \leq 1} \int_{\mathbb{R}}(G * \varphi)(\xi) y_{0}(\xi) d \xi \\
& =\sup _{\|\varphi\|_{L^{\infty}(\mathbb{R})} \leq 1}\|G * \varphi\|_{L^{\infty}(\mathbb{R})}\left\|y_{0}\right\|_{\mathcal{M}(\mathbb{R})} \\
& \leq \sup _{\|\varphi\|_{L^{\infty}(\mathbb{R})} \leq 1}\|G\|_{L^{1}(\mathbb{R})}\|\varphi\|_{L^{\infty}(\mathbb{R})}\left\|y_{0}\right\|_{\mathcal{M}(\mathbb{R})}=\left\|y_{0}\right\|_{\mathcal{M}(\mathbb{R})} .
\end{aligned}
$$

Let us define $u_{0}^{n}:=\rho_{n} * u_{0} \in H^{\infty}(\mathbb{R})$ for $n \geq 1$. Obviously, we get

$$
u_{0}^{n} \rightarrow u_{0} \quad \text { in } H^{1}(\mathbb{R}) \text { for } n \rightarrow \infty
$$


and

$$
\begin{aligned}
& \left\|u_{0}^{n}\right\|_{H^{1}(\mathbb{R})}=\left\|\rho_{n} * u_{0}\right\|_{H^{1}(\mathbb{R})}=\left\|\rho_{n} * u_{0}\right\|_{L^{2}}+\left\|\rho_{n} * u_{0}^{\prime}\right\|_{L^{2}} \leq\left\|u_{0}\right\|_{H^{1}(\mathbb{R})}, \\
& \left\|u_{0}^{n}\right\|_{L^{\infty}(\mathbb{R})}=\left\|G * y_{0}^{n}\right\|_{L^{\infty}(\mathbb{R})} \leq\left\|y_{0}^{n}\right\|_{L^{1}(\mathbb{R})} \leq\left\|y_{0}\right\|_{\mathcal{M}} \\
& \left\|u_{0}^{n}\right\|_{L^{3}(\mathbb{R})}=\left\|\rho_{n} * u_{0}\right\|_{L^{3}(\mathbb{R})} \leq\left\|\rho_{n}\right\|_{L^{1}}\left\|u_{0}\right\|_{L^{3}} \leq\left\|u_{0}\right\|_{L^{3}} .
\end{aligned}
$$

Note that, for all $n \geq 1$,

$$
y_{0}^{n}:=u_{0}^{n}-u_{0, x x}^{n}=\rho_{n} *\left(y_{0}\right) \geq 0 .
$$

Referring to the proof of (9), we have

$$
\left\|y_{0}^{n}\right\|_{L^{1}(\mathbb{R})} \leq\left\|y_{0}\right\|_{\mathcal{M}(\mathbb{R})}, \quad n \geq 1 .
$$

From Lemma 3.1, we know that there exists a global strong solution,

$$
u^{n}=u^{n}\left(\cdot, u_{0}^{n}\right) \in C\left([0, \infty) ; H^{s}(\mathbb{R})\right) \cap C^{1}\left([0, \infty) ; H^{s-1}(\mathbb{R})\right), \quad s \geq \frac{3}{2}
$$

and $u^{n}(t, x)-u_{x x}^{n}(t, x) \geq 0$ for all $(t, x) \in \mathbb{R}_{+} \times \mathbb{R}$.

Note that for all $(t, x) \in \mathbb{R}_{+} \times \mathbb{R}$

$$
\left(u^{n}\right)^{2}=\int_{-\infty}^{x} 2 u^{n} u_{x}^{n} d \xi \leq \int_{\mathbb{R}}\left[\left(u^{n}\right)^{2}+\left(u_{x}^{n}\right)^{2}\right] d \xi=\left\|u^{n}\right\|_{H^{1}}^{2} .
$$

From Lemma 3.1 and (10), we obtain

$$
\begin{aligned}
\left\|u_{x}^{n}\right\|_{L^{\infty}(\mathbb{R})}^{2} & \leq\left\|u^{n}\right\|_{L^{\infty}(\mathbb{R})}^{2} \leq\left\|u^{n}\right\|_{H^{1}(\mathbb{R})}^{2} \\
& \leq\left\|u_{0}^{n}\right\|_{H^{1}}^{2} \exp \left[3\left\|u_{0}^{n}\right\|_{L^{3}}^{2} t^{2}+4\left\|u_{0}^{n}\right\|_{L^{\infty}} t\right] \\
& \leq\left\|u_{0}\right\|_{H^{1}}^{2} \exp \left[3\left\|u_{0}\right\|_{L^{3}}^{2} t^{2}+4\left\|y_{0}\right\|_{\mathcal{M}} t\right] .
\end{aligned}
$$

From the Hölder inequality, Lemma 3.1 and (10), for all $t \geq 0$ and $n \geq 1$, we have

$$
\begin{aligned}
\left\|2 u^{n}(t) u_{x}^{n}(t)\right\|_{L^{2}(\mathbb{R})} & \leq 2\left\|u^{n}(t)\right\|_{L^{\infty}(\mathbb{R})}\left\|u_{x}^{n}(t)\right\|_{L^{2}(\mathbb{R})} \leq 2\left\|u^{n}\right\|_{H^{1}(\mathbb{R})}^{2} \\
& \leq 2\left\|u_{0}^{n}\right\|_{H^{1}}^{2} \exp \left[3\left\|u_{0}^{n}\right\|_{L^{3}}^{2} t^{2}+4\left\|u_{0}^{n}\right\|_{L^{\infty}} t\right] \\
& \leq 2\left\|u_{0}\right\|_{H^{1}}^{2} \exp \left[3\left\|u_{0}\right\|_{L^{3}}^{2} t^{2}+4\left\|y_{0}\right\|_{\mathcal{M}} t\right] .
\end{aligned}
$$

Using the Young inequality, we get

$$
\begin{aligned}
& \left\|\partial_{x} G *\left[\left(u^{n}\right)^{2}+\left(\left(u^{n}\right)^{2}\right)_{x}\right]\right\|_{L^{2}(\mathbb{R})} \\
& \quad \leq\left\|\partial_{x} G *\left(u^{n}\right)^{2}\right\|_{L^{2}(\mathbb{R})}+\left\|\partial_{x} G *\left(u^{n}\right)_{x}^{2}\right\|_{L^{2}(\mathbb{R})} \\
& \quad \leq\left\|\partial_{x} G\right\|_{L^{2}(\mathbb{R})}\left\|\left(u^{n}\right)^{2}\right\|_{L^{1}(\mathbb{R})}+\left\|\partial_{x} G\right\|_{L^{2}(\mathbb{R})}\left\|\left(\left(u^{n}\right)^{2}\right)_{x}\right\|_{L^{1}(\mathbb{R})} \\
& \quad \leq 2\left\|\partial_{x} G\right\|_{L^{2}(\mathbb{R})}\left\|u^{n}\right\|_{H^{1}(\mathbb{R})}^{2}
\end{aligned}
$$




$$
\begin{aligned}
& \leq 2\left\|\partial_{x} G\right\|_{L^{2}(\mathbb{R})}\left\|u_{0}^{n}\right\|_{H^{1}}^{2} \exp \left[3\left\|u_{0}^{n}\right\|_{L^{3}}^{2} t^{2}+4\left\|u_{0}^{n}\right\|_{L^{\infty}} t\right] \\
& \leq 2\left\|\partial_{x} G\right\|_{L^{2}(\mathbb{R})}\left\|u_{0}\right\|_{H^{1}}^{2} \exp \left[3\left\|u_{0}\right\|_{L^{3}}^{2} t^{2}+4\left\|y_{0}\right\|_{\mathcal{M}} t\right]
\end{aligned}
$$

where $\left\|\partial_{x} G\right\|_{L^{2}(\mathbb{R})}$ is bounded.

Applying (13)-(14) and problem (6), we have

$$
\begin{aligned}
& \left\|\frac{d}{d t} u^{n}\right\|_{L^{2}(\mathbb{R})} \\
& \quad \leq 2\left(1+\left\|\partial_{x} G\right\|_{L^{2}(\mathbb{R})}\right)\left\|u_{0}\right\|_{H^{1}}^{2} \exp \left[3\left\|u_{0}\right\|_{L^{3}}^{2} t^{2}+4\left\|y_{0}\right\|_{\mathcal{M}} t\right] .
\end{aligned}
$$

For fixed $T>0$, from (12) and (15), we deduce

$$
\int_{0}^{T} \int_{\mathbb{R}}\left(\left[u^{n}(t, x)\right]^{2}+\left[u_{x}^{n}(t, x)\right]^{2}+\left[u_{t}^{n}(t, x)\right]^{2}\right) d x d t \leq M
$$

where $M$ is a positive constant depending only on $\left\|G_{x}\right\|_{L^{2}(\mathbb{R})},\left\|u_{0}\right\|_{H^{1}(\mathbb{R})},\left\|u_{0}\right\|_{L^{3}(\mathbb{R})},\left\|y_{0}\right\|_{\mathcal{M}(\mathbb{R})}$ and $T$. It follows that the sequence $\left\{u^{n}\right\}_{n \geq 1}$ is uniformly bounded in the space $H^{1}((0$, $T) \times \mathbb{R})$. Thus, we can extract a subsequence such that

$$
u^{n_{k}} \rightarrow u, \quad \text { weakly in } H^{1}((0, T) \times \mathbb{R}) \text { for } n_{k} \rightarrow \infty,
$$

and

$$
u^{n_{k}} \rightarrow u, \quad \text { a.e. on }(0, T) \times \mathbb{R} \text { for } n_{k} \rightarrow \infty,
$$

for some $u \in H^{1}((0, T) \times \mathbb{R})$. From Lemma 3.1 and (10), for fixed $t \in(0, T)$, we see that the sequence $u_{x}^{n_{k}}(t, \cdot) \in \mathrm{BV}(\mathbb{R})$ satisfies

$$
\begin{aligned}
V\left[u_{x}^{n_{k}}(t, x)\right] & =\left\|u_{x x}^{n_{k}}(t, \cdot)\right\|_{L^{1}(\mathbb{R})} \leq\left\|u^{n_{k}}(t, \cdot)\right\|_{L^{1}(\mathbb{R})}+\left\|y^{n_{k}}(t, \cdot)\right\|_{L^{1}(\mathbb{R})} \\
& \leq 2\left\|u_{0}^{n_{k}}(t, \cdot)\right\|_{L^{1}(\mathbb{R})} \leq 2\left\|u_{0}(t, \cdot)\right\|_{L^{1}(\mathbb{R})} \leq 2\left\|y_{0}(t, \cdot)\right\|_{\mathcal{M}(\mathbb{R})}
\end{aligned}
$$

and

$$
\left\|u_{x}^{n_{k}}(t, \cdot)\right\|_{L^{\infty}} \leq\left\|u^{n_{k}}\right\|_{L^{\infty}(\mathbb{R})} \leq\left\|u^{n_{k}}\right\|_{H^{1}(\mathbb{R})} \leq\left\|u_{0}\right\|_{H^{1}(\mathbb{R})} .
$$

Applying Helly's theorem [21], we infer that there exists a subsequence, denoted again $\left\{u_{x}^{n_{k}}(t, \cdot)\right\}$, which converges at every point to some function $v(t, \cdot)$ of finite variation with

$$
V(v(t, \cdot)) \leq 2\left\|y_{0}\right\|_{\mathcal{M}(\mathbb{R})} .
$$

From (18), we get, for almost all $t \in(0, T), u_{x}^{n_{k}}(t, \cdot) \rightarrow u_{x}(t, \cdot)$ in $D^{\prime}(\mathbb{R})$. It follows that $v(t, \cdot)=$ $u_{x}(t, \cdot)$ for a.e. $t \in(0, T)$. Therefore, we have

$$
u_{x}^{n_{k}}(t, \cdot) \rightarrow u_{x}(t, \cdot) \quad \text { a.e. on }(0, T) \times \mathbb{R} \text { for } n_{k} \rightarrow \infty,
$$


and for a.e. $t \in(0, T)$,

$$
V\left[u_{x}(t, \cdot)\right]=\left\|u_{x x}(t, \cdot)\right\|_{\mathcal{M}(\mathbb{R})}=2\left\|u_{0}\right\|_{L^{1}} \leq 2\left\|y_{0}\right\|_{\mathcal{M}(\mathbb{R})}
$$

By Lemma 3.1 and (12), we have

$$
\begin{aligned}
\left\|\left(u^{n}\right)^{2}+\left(\left(u^{n}\right)^{2}\right)_{x}\right\|_{L^{2}(\mathbb{R})} & \leq\left\|\left(u^{n}\right)^{2}\right\|_{L^{2}(\mathbb{R})}+\left\|\left(\left(u^{n}\right)^{2}\right)_{x}\right\|_{L^{2}} \\
& \leq\left\|u^{n}\right\|_{L^{\infty}}\left\|u^{n}\right\|_{L^{2}(\mathbb{R})}+2\left\|u_{x}^{n}\right\|_{L^{\infty}}\left\|u^{n}\right\|_{L^{2}} \\
& \leq\left\|u^{n}\right\|_{H^{1}(\mathbb{R})}^{2}+2\left\|u^{n}\right\|_{H^{1}(\mathbb{R})}^{2} \\
& =3\left\|u^{n}\right\|_{H^{1}(\mathbb{R})}^{2} \\
& \leq 3\left\|u_{0}\right\|_{H^{1}}^{2} \exp \left[3\left\|u_{0}\right\|_{L^{3}}^{2} t^{2}+4\left\|y_{0}\right\|_{\mathcal{M}} t\right] .
\end{aligned}
$$

Note that, for fixed $t \in(0, T)$, the sequence $\left\{\left(u^{n}\right)^{2}+\left(\left(u^{n}\right)^{2}\right)_{x}\right\}_{n \geq 1}$ is uniformly bounded in $L^{2}(\mathbb{R})$. Therefore, it has a subsequence $\left\{\left(u^{n_{k}}\right)^{2}+\left(\left(u^{n_{k}}\right)^{2}\right)_{x}\right\}_{n_{k} \geq 1}$ which converges weakly in $L^{2}(\mathbb{R})$. From (18), we infer that the weak $L^{2}(\mathbb{R})$-limit is $\left\{(u)^{2}+\left(u^{2}\right)_{x}\right\}$. It follows from $G_{x} \in L^{2}(\mathbb{R})$ that

$$
\partial_{x} G *\left(\left(u^{n_{k}}\right)^{2}+\left(\left(u^{n_{k}}\right)^{2}\right)_{x}\right) \rightarrow \partial_{x} G *\left(u^{2}+\left(u^{2}\right)_{x}\right) \quad \text { for } n_{k} \rightarrow \infty
$$

From (18), (19) and (21), we see that $u$ solves Eq. (6) in $D^{\prime}((0, T) \times \mathbb{R})$.

For fixed $T>0$, noticing that $u_{t}^{n_{k}}$ is uniformly bounded in $L^{2}(\mathbb{R})$ as $t \in[0, T)$ and $\left\|u^{n_{k}}(t)\right\|_{H^{1}(\mathbb{R})}$ is uniformly bounded for all $t \in[0, T)$ and $n \geq 1$, we infer that the family $t \rightarrow u^{n_{k}} \in H^{1}(\mathbb{R})$ is weakly equicontinuous on $[0, \mathrm{~T}]$. An application of the Arzela-Ascoli theorem shows that $\left\{u^{n_{k}}\right\}$ has a subsequence, denoted again $\left\{u^{n_{k}}\right\}$, which converges weakly in $H^{1}(\mathbb{R})$, uniformly in $t \in[0, T)$. The limit function is $u$. T being arbitrary, we see that $u$ is locally and weakly continuous from $[0, \infty)$ into $H^{1}(\mathbb{R})$, i.e., $u \in C_{w, \text { loc }}\left(\mathbb{R}_{+} ; H^{1}(\mathbb{R})\right.$ ).

Since for a.e. $t \in \mathbb{R}_{+}, u^{n_{k}}(t, \cdot) \rightarrow u(t, \cdot)$ weakly in $H^{1}(\mathbb{R})$, from Lemma 3.1, we get

$$
\begin{aligned}
\|u(t, \cdot)\|_{L^{\infty}(\mathbb{R})} & \leq\|u(t, \cdot)\|_{H^{1}(\mathbb{R})} \leq \liminf _{n_{k} \rightarrow \infty}\left\|u^{n_{k}}(t, \cdot)\right\|_{H^{1}(\mathbb{R})} \\
& \leq\left\|u_{0}\right\|_{H^{1}} \exp \left[3\left\|u_{0}\right\|_{L^{3}}^{2} t^{2}+4\left\|y_{0}\right\|_{\mathcal{M}} t\right] .
\end{aligned}
$$

Inequality (22) shows that

$$
u \in L_{\mathrm{loc}}^{\infty}\left(\mathbb{R}_{+} \times \mathbb{R}\right) \cap L_{\mathrm{loc}}^{\infty}\left(\mathbb{R}_{+} ; H^{1}(\mathbb{R})\right) .
$$

From (10), for $t \in \mathbb{R}_{+}$, we obtain

$$
\left\|u_{x}^{n}(t, \cdot)\right\|_{L^{\infty}} \leq\left\|u^{n}\right\|_{L^{\infty}(\mathbb{R})} \leq\left\|u_{0}\right\|_{H^{1}(\mathbb{R})} .
$$

Combining with (18), we have

$$
u_{x} \in L^{\infty}\left(\mathbb{R}_{+} \times \mathbb{R}\right)
$$


Finally, we prove $\left(u(t, \cdot)-u_{x x}(t, \cdot)\right) \in \mathcal{M}^{+}$is uniformly bounded on $\mathbb{R}$ and $u(t, x) \in$ $W^{1, \infty}\left(\mathbb{R}_{+} \times \mathbb{R}\right)$.

We have

$$
L^{1}(\mathbb{R}) \subset\left(L^{\infty}\right)^{*} \subset\left(C_{0}(\mathbb{R})\right)^{*}=\mathcal{M}(\mathbb{R})
$$

From (20), we get for a.e. $t \in \mathbb{R}_{+}$

$$
\begin{aligned}
\left\|u(t, \cdot)-u_{x x}(t, \cdot)\right\|_{\mathcal{M}(\mathbb{R})} & \leq\|u(t, \cdot)\|_{L^{1}(\mathbb{R})}+\left\|u_{x x}(t, \cdot)\right\|_{\mathcal{M}(\mathbb{R})} \\
& \leq\left\|u_{0}\right\|_{L^{1}(\mathbb{R})}+2\left\|y_{0}\right\|_{\mathcal{M}(\mathbb{R})} \leq 3\left\|y_{0}\right\|_{\mathcal{M}(\mathbb{R})} .
\end{aligned}
$$

The above inequality implies that, for a.e. $t \in \mathbb{R}_{+},\left(u(t, \cdot)-u_{x x}(t, \cdot)\right) \in \mathcal{M}(\mathbb{R})$ is uniformly bounded on $\mathbb{R}$. For fixed $T \geq 0$, applying (17) and (18), we have

$$
\left[u^{n_{k}}(t, \cdot)-u_{x x}^{n_{k}}(t, \cdot)\right] \rightarrow\left[u(t, \cdot)-u_{x x}(t, \cdot)\right] \quad \text { in } D^{\prime}(\mathbb{R}) \text { for } n \rightarrow \infty
$$

Since $\left(u^{n_{k}}(t, \cdot)-u_{x x}^{n_{k}}(t, \cdot)\right) \geq 0$ for all $(t, x) \in \mathbb{R}_{+} \times \mathbb{R}$, we obtain, for a.e. $t \in \mathbb{R}_{+},(u(t, \cdot)-$ $\left.u_{x x}(t, \cdot)\right) \in \mathcal{M}^{+}(\mathbb{R})$.

Note that $u(t, x)=G *\left(u(t, x)-u_{x x}(t, x)\right)$. Then we get

$$
\begin{aligned}
|u(t, x)| & =\left|G *\left(u(t, x)-u_{x x}(t, x)\right)\right| \leq\|G\|_{L^{\infty}(\mathbb{R})}\left\|u(t, x)-u_{x x}(t, x)\right\|_{\mathcal{M}(\mathbb{R})} \\
& \leq 3\left\|y_{0}\right\|_{\mathcal{M}(\mathbb{R}) .}
\end{aligned}
$$

Combining with (23), it implies that $u(t, x) \in W^{1, \infty}\left(\mathbb{R}_{+} \times \mathbb{R}\right)$.

This completes the proof of the existence of Theorem 3.1.

Next, we present the uniqueness proof of Theorem 3.1.

Let $u, v \in W^{1, \infty}\left(\mathbb{R}_{+} \times \mathbb{R}\right) \cap L_{\text {loc }}^{\infty}\left(\mathbb{R}_{+} ; H^{1}(\mathbb{R})\right)$ be two global weak solutions of problem (6) with the same initial data $u_{0}$. Assume that $\left(u(t, \cdot)-u_{x x}(t, \cdot)\right) \in \mathcal{M}^{+}(\mathbb{R})$ and $\left(v(t, \cdot)-v_{x x}(t, \cdot)\right) \in$ $\mathcal{M}^{+}(\mathbb{R})$ are uniformly bounded on $\mathbb{R}_{+}$and set

$$
N:=\sup _{t \in \mathbb{R}_{+}}\left\{\left\|u(t, \cdot)-u_{x x}(t, \cdot)\right\|_{\mathcal{M}(\mathbb{R})}+\left\|v(t, \cdot)-v_{x x}(t, \cdot)\right\|_{\mathcal{M}(\mathbb{R})}\right\} .
$$

From the assumption, we know that $N<\infty$. Then, for all $(t, x) \in \mathbb{R}_{+} \times \mathbb{R}$,

$$
\begin{aligned}
|u(t, x)| & =\left|G *\left(u(t, x)-u_{x x}(t, x)\right)\right| \\
& \leq\|G\|_{L^{\infty}(\mathbb{R})}\left\|u(t, x)-u_{x x}(t, x)\right\|_{\mathcal{M}(\mathbb{R})} \leq \frac{N}{2}
\end{aligned}
$$

and

$$
\begin{aligned}
\left|u_{x}(t, x)\right| & =\left|G_{x} *\left(u(t, x)-u_{x x}(t, x)\right)\right| \\
& \leq\left\|G_{x}\right\|_{L^{\infty}(\mathbb{R})}\left\|u(t, x)-u_{x x}(t, x)\right\|_{\mathcal{M}(\mathbb{R})} \leq \frac{N}{2} .
\end{aligned}
$$

Similarly

$$
|v(t, x)| \leq \frac{N}{2}, \quad\left|v_{x}(t, x)\right| \leq \frac{N}{2}, \quad(t, x) \in \mathbb{R}_{+} \times \mathbb{R} .
$$


Following the same procedure as in (9), we may also get

$$
\begin{aligned}
&\|u(t, x)\|_{L^{1}}=\left\|G *\left(u(t, x)-u_{x x}(t, x)\right)\right\|_{L^{1}(\mathbb{R})} \\
& \leq\|G\|_{L^{1}(\mathbb{R})}\left\|u(t, x)-u_{x x}(t, x)\right\|_{\mathcal{M}(\mathbb{R})} \leq N \\
&\left\|u_{x}(t, x)\right\|_{L^{1}(\mathbb{R})}=\left\|G_{x} *\left(u(t, x)-u_{x x}(t, x)\right)\right\|_{L^{1}(\mathbb{R})} \\
& \leq\left\|G_{x}\right\|_{L^{1}(\mathbb{R})}\left\|u(t, x)-u_{x x}(t, x)\right\|_{\mathcal{M}(\mathbb{R})} \leq N,
\end{aligned}
$$

and for all $(t, x) \in \mathbb{R}_{+} \times \mathbb{R}$

$$
\|v(t, x)\|_{L^{1}(\mathbb{R})} \leq N, \quad\left\|v_{x}(t, x)\right\|_{L^{1}(\mathbb{R})} \leq N .
$$

We define

$$
w(t, x):=u(t, x)-v(t, x), \quad(t, x) \in \mathbb{R}_{+} \times \mathbb{R} .
$$

Convoluting Eq. (6) for $u$ and $v$ with $\rho_{n}$, we get, for a.e. $t \in \mathbb{R}_{+}$and all $n \geq 1$,

$$
\rho_{n} * u_{t}+\rho_{n} *\left(-2 u u_{x}\right)+\rho_{n} * \partial_{x} G *\left[u^{2}+\left(u^{2}\right)_{x}\right]=0
$$

and

$$
\rho_{n} * v_{t}+\rho_{n} *\left(-2 v v_{x}\right)+\rho_{n} * \partial_{x} G *\left[v^{2}+\left(v^{2}\right)_{x}\right]=0
$$

Subtracting (31) from (30) and using Lemma 2.4, integration by parts shows that for a.e. $t \in \mathbb{R}_{+}$and all $n \geq 1$

$$
\begin{aligned}
\frac{d}{d t} \int_{\mathbb{R}}\left|\rho_{n} * w\right| d x= & \int_{\mathbb{R}}\left(\rho_{n} * w_{t}\right) \operatorname{sgn}\left(\rho_{n} * w\right) d x \\
= & 2 \int_{\mathbb{R}}\left(\rho_{n} * w u_{x}\right) \operatorname{sgn}\left(\rho_{n} * w\right) d x \\
& +2 \int_{\mathbb{R}}\left(\rho_{n} * v w_{x}\right) \operatorname{sgn}\left(\rho_{n} * w\right) d x \\
& +\int_{\mathbb{R}}\left(\rho_{n} * \partial_{x} G * w(u+v)\right) \operatorname{sgn}\left(\rho_{n} * w\right) d x \\
& -2 \int_{\mathbb{R}}\left(\rho_{n} * \partial_{x} G * w_{x} u\right) \operatorname{sgn}\left(\rho_{n} * w\right) d x \\
& -2 \int_{\mathbb{R}}\left(\rho_{n} * \partial_{x} G * w v_{x}\right) \operatorname{sgn}\left(\rho_{n} * w\right) d x .
\end{aligned}
$$

Using (24)-(26) and the Young inequality to the first term on the right-hand of (32) yields

$$
\begin{aligned}
& \left|\int_{\mathbb{R}}\left(\rho_{n} *\left(w u_{x}\right)\right) \operatorname{sgn}\left(\rho_{n} * w\right) d x\right| \\
& \quad \leq \int_{\mathbb{R}}\left|\left(\rho_{n} *\left(w u_{x}\right)\right)\right| d x
\end{aligned}
$$




$$
\begin{aligned}
\leq & \int_{\mathbb{R}}\left|\rho_{n} * w\right|\left|\rho_{n} * u_{x}\right| d x+\int_{\mathbb{R}}\left|\rho_{n} *\left(w u_{x}\right)-\left(\rho_{n} * w\right)\left(\rho_{n} * u_{x}\right)\right| d x \\
\leq & \left\|\rho_{n} * u_{x}\right\|_{L^{\infty}} \int_{\mathbb{R}}\left|\rho_{n} * w\right| d x \\
& +\int_{\mathbb{R}}\left|\rho_{n} *\left(w u_{x}\right)-\left(\rho_{n} * w\right)\left(\rho_{n} * u_{x}\right)\right| d x \\
\leq & \left\|\rho_{n}\right\|_{L^{1}}\left\|u_{x}\right\|_{L^{\infty}} \int_{\mathbb{R}}\left|\rho_{n} * w\right| d x \\
& +\int_{\mathbb{R}}\left|\rho_{n} *\left(w u_{x}\right)-\left(\rho_{n} * w\right)\left(\rho_{n} * u_{x}\right)\right| d x \\
\leq & \frac{N}{2} \int_{\mathbb{R}}\left|\rho_{n} * w\right| d x+\int_{\mathbb{R}}\left|\rho_{n} *\left(w u_{x}\right)-\left(\rho_{n} * w\right)\left(\rho_{n} * u_{x}\right)\right| d x .
\end{aligned}
$$

Similarly, we obtain

$$
\begin{aligned}
& \left|\int_{\mathbb{R}}\left(\rho_{n} *\left(w_{x} v\right)\right) \operatorname{sgn}\left(\rho_{n} * w\right) d x\right| \\
& \quad \leq \int_{\mathbb{R}}\left|\left(\rho_{n} *\left(w_{x} v\right)\right)\right| d x \\
& \quad \leq \int_{\mathbb{R}}\left|\rho_{n} * w_{x}\right|\left|\rho_{n} * v\right| d x+\int_{\mathbb{R}}\left|\rho_{n} *\left(w_{x} v\right)-\left(\rho_{n} * w_{x}\right)\left(\rho_{n} * v\right)\right| d x \\
& \quad \leq \frac{N}{2} \int_{\mathbb{R}}\left|\rho_{n} * w_{x}\right| d x+\int_{\mathbb{R}}\left|\rho_{n} *\left(w_{x} v\right)-\left(\rho_{n} * w_{x}\right)\left(\rho_{n} * v\right)\right| d x
\end{aligned}
$$

and

$$
\begin{aligned}
\left|\int_{\mathbb{R}}\left(\rho_{n} * \partial_{x} G *[w(u+v)]\right) \operatorname{sgn}\left(\rho_{n} * w\right) d x\right| \\
\leq \int_{\mathbb{R}} \mid\left(\rho_{n} * G *\left[w_{x}(u+v)\right] \mid d x\right. \\
\quad+\int_{\mathbb{R}} \mid\left(\rho_{n} * G *\left[w(u+v)_{x}\right] \mid d x\right. \\
\leq \frac{1}{2} \int_{\mathbb{R}}\left|\rho_{n} *\left[w_{x}(u+v)\right]\right| d x+\frac{1}{2} \int_{\mathbb{R}}\left|\rho_{n} *\left[w\left(u_{x}+v_{x}\right)\right]\right| d x \\
\leq \frac{N}{2} \int_{\mathbb{R}}\left|\rho_{n} * w_{x}\right| d x+\frac{N}{2} \int_{\mathbb{R}}\left|\rho_{n} * w\right| d x \\
\quad+\int_{\mathbb{R}}\left|\rho_{n} *\left(w_{x}(u+v)\right)-\left(\rho_{n} * w_{x}\right)\left[\rho_{n} *(u+v)\right]\right| d x \\
\quad+\int_{\mathbb{R}}\left|\rho_{n} *\left[w(u+v)_{x}\right]-\left(\rho_{n} * w\right)\left[\rho_{n} *(u+v)_{x}\right]\right| d x .
\end{aligned}
$$

For the last term on the right-hand side of (32), we have

$$
\begin{aligned}
& \left|\int_{\mathbb{R}}\left(\rho_{n} * \partial_{x} G *\left(w_{x} u\right)\right) \operatorname{sgn}\left(\rho_{n} * w\right) d x\right| \\
& \quad \leq \int_{\mathbb{R}}\left|\rho_{n} * \partial_{x} G *\left(w_{x} u\right)\right| d x
\end{aligned}
$$




$$
\begin{aligned}
& \leq\left\|\partial_{x} G\right\|_{L^{1}(\mathbb{R})} \int_{\mathbb{R}}\left|\rho_{n} *\left(w_{x} u\right)\right| d x \\
& \leq N \int_{\mathbb{R}}\left|\rho_{n} * w_{x}\right| d x+\int_{\mathbb{R}}\left(\left|\rho_{n} *\left(w_{x} u\right)-\left(\rho_{n} * w_{x}\right)\left(\rho_{n} * u\right)\right| d x\right.
\end{aligned}
$$

and

$$
\begin{aligned}
& \left|\int_{\mathbb{R}}\left(\rho_{n} * \partial_{x} G *\left(w v_{x}\right)\right) \operatorname{sgn}\left(\rho_{n} * w\right) d x\right| \\
& \quad \leq \int_{\mathbb{R}}\left|\rho_{n} * \partial_{x} G *\left(w v_{x}\right)\right| d x \\
& \quad \leq\left\|\partial_{x} G\right\|_{L^{1}(\mathbb{R})} \int_{\mathbb{R}}\left|\rho_{n} *\left(w v_{x}\right)\right| d x \\
& \quad \leq N \int_{\mathbb{R}}\left|\rho_{n} * w\right| d x+\int_{\mathbb{R}}\left|\rho_{n} *\left(w v_{x}\right)-\left(\rho_{n} * w\right)\left(\rho_{n} * v_{x}\right)\right| d x
\end{aligned}
$$

From (33)-(37), for a.e. $t \in \mathbb{R}_{+}$and all $n \geq 1$, we find

$$
\begin{aligned}
\frac{d}{d t} \int_{\mathbb{R}}\left|\rho_{n} * w\right| d x \leq & 2 N \int_{\mathbb{R}}\left|\rho_{n} * w\right| d x \\
& +2 N \int_{\mathbb{R}}\left|\rho_{n} * w_{x}\right| d x+R_{n}(t),
\end{aligned}
$$

where

$$
\begin{cases}R_{n}(t) \rightarrow 0 & \text { as } t \rightarrow \infty \\ \left|R_{n}(t)\right| \leq K, & n \geq 1, t \in \mathbb{R}_{+}\end{cases}
$$

where $K$ is a positive constant depending on $N$ and the $H^{1}(\mathbb{R})$-norms of $u(0)$ and $v(0)$.

In the same way, convoluting Eq. (6) for $u$ and $v$ with $\rho_{n, x}$ and using Lemma 2.4, we see that for a.e. $t \in \mathbb{R}_{+}$and all $n \geq 1$

$$
\begin{aligned}
\frac{d}{d t} \int_{\mathbb{R}}\left|\rho_{n} * w_{x}\right| d x= & \int_{\mathbb{R}}\left(\rho_{n} * w_{x t}\right) \operatorname{sgn}\left(\rho_{n, x} * w\right) d x \\
= & 2 \int_{\mathbb{R}}\left(\rho_{n} * w_{x}\left(u_{x}+v_{x}\right)\right) \operatorname{sgn}\left(\rho_{n, x} * w\right) d x \\
& +2 \int_{\mathbb{R}}\left(\rho_{n} * v_{x x} w\right) \operatorname{sgn}\left(\rho_{n, x} * w\right) d x \\
& +2 \int_{\mathbb{R}}\left(\rho_{n} * u w_{x x}\right) \operatorname{sgn}\left(\rho_{n, x} * w\right) d x \\
& -\int_{\mathbb{R}}\left(\rho_{n} * \partial_{x x} G *\left[\left(u^{2}-v^{2}\right)+\left(u^{2}\right)_{x}-\left(v^{2}\right)_{x}\right] \operatorname{sgn}\left(\rho_{n, x} * w\right) d x . \quad(40)\right.
\end{aligned}
$$

Using the identity $\partial_{x}^{2}(G * g)=G * g-g$ for $g \in L^{2}(\mathbb{R})$ and the Young inequality, we estimate the fourth term of the right-hand side of (40):

$$
\begin{aligned}
& \mid \int_{\mathbb{R}}\left(\rho_{n} * \partial_{x x} G *\left[\left(u^{2}-v^{2}\right)+\left(u^{2}\right)_{x}-\left(v^{2}\right)_{x}\right] d x \mid\right. \\
& \quad \leq \int_{\mathbb{R}} \mid\left(\rho_{n} * G *\left[\left(u^{2}-v^{2}\right)+\left(u^{2}\right)_{x}-\left(v^{2}\right)_{x}\right] \mid d x\right.
\end{aligned}
$$




$$
\begin{aligned}
& +\int_{\mathbb{R}} \mid\left(\rho_{n} *\left[\left(u^{2}-v^{2}\right)+\left(u^{2}\right)_{x}-\left(v^{2}\right)_{x}\right] \mid d x\right. \\
\leq & \left(\|G\|_{L^{1}(\mathbb{R})}+1\right) \int_{\mathbb{R}} \mid\left(\rho_{n} *\left[w(u+v)+2 u w_{x}+2 w v_{x}\right] \mid d x\right. \\
\leq & 2 \int_{\mathbb{R}} \mid\left(\rho_{n} *[w(u+v)]\left|d x+4 \int_{\mathbb{R}}\right|\left(\rho_{n} *\left[u w_{x}+w v_{x}\right)\right] \mid d x\right. \\
\leq & 4 N \int_{\mathbb{R}}\left|\rho_{n} * w\right| d x+2 N \int_{\mathbb{R}}\left|\rho_{n} * w_{x}\right| d x+R_{n} .
\end{aligned}
$$

Using (24)-(26) and the Young inequality to the first term on the right-hand of (40) gives rise to

$$
\begin{aligned}
& 2 \int_{\mathbb{R}}\left(\rho_{n} * w_{x}\left(u_{x}+v_{x}\right)\right) \operatorname{sgn}\left(\rho_{n, x} * w\right) d x \\
& \leq 2 \int_{\mathbb{R}}\left|\rho_{n} * w_{x}\left(u_{x}+v_{x}\right)\right| d x \\
& \leq 2 \int_{\mathbb{R}}\left|\rho_{n} * w_{x}\right|\left|\rho_{n} *\left(u_{x}+v_{x}\right)\right| d x \\
& \quad+2 \int_{\mathbb{R}}\left|\rho_{n} * w_{x}\left(u_{x}+v_{x}\right)-\left(\rho_{n} * w_{x}\right)\left(\rho_{n} *\left(u_{x}+v_{x}\right)\right)\right| d x \\
& \leq 2 N \int_{\mathbb{R}}\left|\rho_{n} * w_{x}\right| d x+R_{n} .
\end{aligned}
$$

To treat the second term of the right-hand side of (40), we note that

$$
\begin{aligned}
\left|2 \int_{\mathbb{R}}\left(\rho_{n} * v_{x x} w\right) \operatorname{sgn}\left(\rho_{n, x} * w\right) d x\right| \leq & 2 \int_{\mathbb{R}}\left|\left(\rho_{n} * w\right)\left(\rho_{n} * v_{x x}\right)\right| d x \\
& +2 \int_{\mathbb{R}}\left|\left(\rho_{n} * v_{x x} w\right)-\left(\rho_{n} * w\right)\left(\rho_{n} * v_{x x}\right)\right| d x
\end{aligned}
$$

Applying Lemma 2.1, the second expression of right-hand of (43) can be estimated by a function $R_{n}(t)$ belonging to (39). Making use of the Hölder inequality and (9), for a.e. $t \in \mathbb{R}_{+}$and all $n \geq 1$, we have

$$
\begin{aligned}
\int_{\mathbb{R}}\left|\left(\rho_{n} * w\right)\left(\rho_{n} * v_{x x}\right)\right| d x & \leq\left\|\rho_{n} * w\right\|_{L^{\infty}(\mathbb{R})}\left\|\rho_{n} * v_{x x}\right\|_{L^{1}(\mathbb{R})} \\
& \leq\left\|\rho_{n} * w\right\|_{W^{1,1}(\mathbb{R})}\left\|v_{x x}\right\|_{\mathcal{M}(\mathbb{R})}
\end{aligned}
$$

It follows from (43) and (44) that

$$
\begin{aligned}
\left|2 \int_{\mathbb{R}}\left(\rho_{n} * v_{x x} w\right) \operatorname{sgn}\left(\rho_{n, x} * w\right) d x\right| \leq & 2 N \int_{\mathbb{R}}\left|\rho_{n} * w\right| d x \\
& +2 N \int_{\mathbb{R}}\left|\rho_{n} * w_{x}\right|+R_{n}(t) .
\end{aligned}
$$

Now, we deal with the third term on the right-hand side of (40)

$$
\begin{aligned}
& 2 \int_{\mathbb{R}}\left(\rho_{n} * u w_{x x}\right) \operatorname{sgn}\left(\rho_{n, x} * w\right) d x \\
& \quad=2 \int_{\mathbb{R}}\left(\rho_{n} * u\right)\left(\rho_{n} * w_{x x}\right) \operatorname{sgn}\left(\rho_{n, x} * w\right) d x
\end{aligned}
$$




$$
\begin{aligned}
& +2 \int_{\mathbb{R}}\left[\left(\rho_{n} * u w_{x x}\right)-\left(\rho_{n} * u\right)\left(\rho_{n} * w_{x x}\right)\right] \operatorname{sgn}\left(\rho_{n, x} * w\right) d x \\
\leq & 2 \int_{\mathbb{R}}\left(\rho_{n} * u\right) \frac{\partial}{\partial_{x}}\left|\rho_{n} * w_{x}\right| d x \\
& +2 \int_{\mathbb{R}}\left|\left(\rho_{n} * u w_{x x}\right)-\left(\rho_{n} * u\right)\left(\rho_{n} * w_{x x}\right)\right| d x \\
= & 2 \int_{\mathbb{R}}\left(\rho_{n} * u_{x}\right)\left|\rho_{n} * w_{x}\right| d x+R_{n} .
\end{aligned}
$$

Therefore, (46) implies that for a.e. $t \in \mathbb{R}_{+}$and all $n \geq 1$

$$
\left|2 \int_{\mathbb{R}}\left(\rho_{n} * u w_{x x}\right) \operatorname{sgn}\left(\rho_{n, x} * w\right) d x\right| \leq N \int_{\mathbb{R}}\left|\rho_{n} * w_{x}\right| d x+R_{n} .
$$

From (41), (42), (45) and (47), for a.e. $t \in \mathbb{R}_{+}$and all $n \geq 1$, we deduce

$$
\begin{aligned}
\frac{d}{d t} \int_{\mathbb{R}}\left|\rho_{n} * w_{x}\right| d x \leq & 6 N \int_{\mathbb{R}}\left|\rho_{n} * w\right| d x \\
& +7 N \int_{\mathbb{R}}\left|\rho_{n} * w_{x}\right| d x+R_{n} .
\end{aligned}
$$

Combining with (38) and (48), we find

$$
\begin{aligned}
\frac{d}{d t} \int_{\mathbb{R}}\left(\left|\rho_{n} * w\right|+\left|\rho_{n} * w_{x}\right|\right) d x \leq & 8 N \int_{\mathbb{R}}\left|\rho_{n} * w\right| d x \\
& +9 N \int_{\mathbb{R}}\left|\rho_{n} * w_{x}\right| d x+R_{n} \\
\leq & 9 N \int_{\mathbb{R}}\left(\left|\rho_{n} * w\right|+\left|\rho_{n} * w_{x}\right|\right) d x+R_{n} .
\end{aligned}
$$

It follows from the Gronwall inequality that for a.e. $t \in \mathbb{R}_{+}$and all $n \geq 1$

$$
\begin{aligned}
\int_{\mathbb{R}}\left(\left|\rho_{n} * w\right|+\left|\rho_{n} * w_{x}\right|\right) d x \leq & {\left[\int_{0}^{t} R_{n}(s) d s\right.} \\
& \left.+\int_{\mathbb{R}}\left(\left|\rho_{n} * w\right|+\left|\rho_{n} * w_{x}\right|\right)(0, x) d x\right] e^{9 N t}
\end{aligned}
$$

Fix $t>0$ and let $n \rightarrow \infty$ in (50). Since $w=u-v \in W^{1,1}(\mathbb{R})$ and Eq. (39) holds, making use of Lebesgue's dominated convergence theorem yields

$$
\int_{\mathbb{R}}\left(|w|+\left|w_{x}\right|\right) d x \leq\left[\int_{\mathbb{R}}\left(|w|+\left|w_{x}\right|\right)(0, x) d x\right] e^{9 N t} .
$$

Note that $w(0)=w_{x}(0)=0$, therefore, we obtain $u(t, x)=v(t, x)$ for a.e. $(t, x) \in \mathbb{R}_{+} \times \mathbb{R}$. This completes the proof of the theorem. 
Funding

There is no funding to our work.

\section{Availability of data and materials}

Not applicable.

\section{Competing interests}

The author declares that there is no conflict of interests regarding the publication of this paper

Authors' contributions

The author prepared the manuscript independently, and read and approved the final manuscript.

\section{Publisher's Note}

Springer Nature remains neutral with regard to jurisdictional claims in published maps and institutional affiliations.

\section{Received: 7 September 2019 Accepted: 13 January 2020 Published online: 23 January 2020}

\section{References}

1. Camassa, R., Holm, D.: An integrable shallow water wave equation with peaked solitons. Phys. Rev. Lett. 71, 1661-1664 (1993)

2. Chen, R.M., Guo, F., Liu, Y., Qu, C.Z.: Analysis on the blow-up of solutions to a class of integrable peakon equations. J. Funct. Anal. 270, 2343-2374 (2016)

3. Coclite, G.M., Holden, H., Karlsen, K.H.: Well-posedness of a parabolic-elliptic system. Discrete Contin. Dyn. Syst. 13 659-682 (2005)

4. Coclite, G.M., Karlsen, K.H.: On the well-posedness of the Degasperis-Procesi equations. J. Funct. Anal. 233, 60-91 (2006)

5. Coclite, G.M. Karlsen, K.H.: Periodic solutions of the Degasperis-Procesi eqution: well-posedness and asymptotics. J. Funct. Anal. 268, 1053-1077 (2015)

6. Constantin, A.: Existence of permanent and breaking waves for a shallow water wave equation: a geometric approach. Ann. Inst. Fourier (Grenoble) 50, 321-362 (2000)

7. Constantin, A., Escher, J.: Global existence and blow-up for a shallow water equation. Ann. Sc. Norm. Super. Pisa 26, 303-328 (1998)

8. Constantin, A., Escher, J.: Wave breaking for nonlinear nonlocal shallow water equations. Acta Math. 181, 229-243 (1998)

9. Constantin, A., Lannes, D.: The hydro-dynamical relevance of the Camassa-Holm and Degasperis-Procesi equations. Arch. Ration. Mech. Anal. 193, 165-186 (2009)

10. Constantin, A., Mckean, H.P.: A shallow water equation on the circle. Commun. Pure Appl. Math. 52, 949-982 (1999)

11. Constantin, A., Molinet, L.: Global weak solution for a shallow water equation. Commun. Math. Phys. 211, 45-61 (2000)

12. Degasperis, A., Holm, D.D., Hone, A.N.W.: A new integrable equation with peakon solution. Theor. Math. Phys. 133, 1463-1474 (2002)

13. Escher, J., Liu, Y., Yin, Z: Shock waves and blow-up phenomena for the periodic Degasperis-Procesi equation. Indiana Univ. Math. J. 56(1), 87-117 (2007)

14. Fuchssteiner, B.: Some tricks from the symmetry-toolbox for nonlinear equations: generalizations of the Camassa-Holm equation. Physica D 95, 229-243 (1996)

15. Gui, G., Liu, Y., Olver, P., Qu, C.: Wave-breaking and peakons for a modified Camassa-Holm equation. Commun. Math. Phys. 319, 731-759 (2013)

16. Lenells, J.: Traveling wave solutions of the Degasperis-Procesi eqaution. J. Math. Anal. Appl. 306, 72-82 (2005)

17. Li, J., Yin, Z: Well-poseness and global existence for a generalized Degasperis-Procesi equation. Nonlinear Anal., Real World Appl. 28, 72-92 (2016)

18. Liu, X., Liu, Y., Qu, C.: Orbital stability of the train of peakons for an integrable modified Camassa-Holm equation. Adv. Math. 255(2), 1-37 (2014)

19. Lundmark, H.: Formation and dynamics of shock waves in the Degasperis-Procesi equation. J. Nonlinear Sci. 17(3), 169-198 (2007)

20. Matsuno, Y: Multisolution solutions of Degasperis-Procesi equation and their peakon limit. Inverse Probl. 21, 1553-1570 (2005)

21. Natanson, I.P.: Theory of Functions of a Real Variable. Ungar, New York (1964)

22. Novikov, V:: Generalizations of the Camassa-Holm equation. J. Phys. A 42, 342002 (2009)

23. Qiao, Z., Li, X.Q.: An integrable equation with nonsmooth solitons. Theor. Math. Phys. 267, 584-589 (2011)

24. Yin, Z:: On the Cauchy problem for an integrable equation with peakon solutions. III. J. Math. 47, 649-666 (2003)

25. Yin, Z:: Global existence for a new periodic integrable equation. J. Math. Anal. Appl. 283, 129-139 (2003)

26. Yin, Z:: Global solutions to a new integrable equation with peakons. Indiana Univ. Math. J. 53(4), 1189-1209 (2004) 